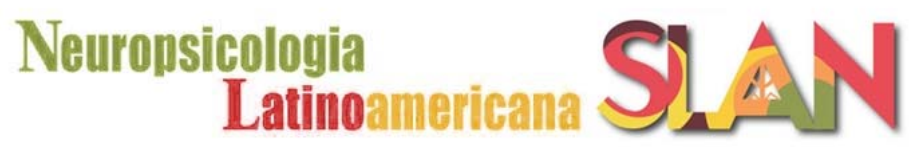

Revista Neuropsicologia Latinoamericana

ISSN 2075-9479 Vol 3. No. 2. 2011, 23-30.

\title{
Correlaciones entre praxias y memoria de trabajo en demencia frontotemporal variante frontal
}

\author{
Corrélations entre les praxies et la mémoire de travail dans la variante frontale de la démence frontotemporale \\ Correlações entre praxia e memória de trabalho na variante frontal da demência frontotemporal \\ Correlations between praxis and working memory in the frontal variant of frontotemporal dementia
}

\section{Pablo G. Gómez ${ }^{1} \&$ Daniel G. Politis ${ }^{1}$}

${ }^{1}$ Hospital Interzonal General de Agudos Eva Perón, Argentina.

Agradecimientos: Este trabajo fue realizado en el marco de los subsidios UBACyT P037 y CONICET PIP 112-200801-00633.

\section{Resumen}

Se ha reportado que una alteración en la memoria de trabajo podría afectar el rendimiento práxico. Los objetivos de este estudio son evaluar en pacientes con demencia frontotemporal variante frontal la presencia de apraxia, valorar la existencia de correlaciones entre apraxia y memoria de trabajo y buscar evidencias de una memoria de trabajo específica para gestos. Material y métodos: Se administraron a 23 pacientes con diagnóstico de demencia fronto-temporal variante frontal la batería cognitiva de evaluación de praxias y dos pruebas de memoria de trabajo. Resultados: Se hallaron correlaciones entre el rendimiento en las pruebas de praxias y las medidas de memoria de trabajo. El rendimiento en memoria de trabajo gestual se mostró disociado respecto del rendimiento en memoria de trabajo visual y verbal. Conclusiones: Estos resultados reafirmarían la importancia de la memoria de trabajo en la producción de praxias y señalarían la posibilidad de una memoria de trabajo específica de gestos.

Palabras clave: Apraxia; demencia frontotemporal variante frontal; memoria de trabajo; memoria visual; memoria gestual.

\section{Résumé}

Il a été décrit dans la littérature qu'une limitation de la mémoire de travail peut affecter les performances praxiques. L'objet de cet étude est d'évaluer la présence d'apraxie chez des patients présentant la variante frontale de la démence frontotemporale, afin de déterminer la présence de corrélations entre l'apraxie et la mémoire de travail, ainsi que de rapporter les preuves d'une mémoire de travail dédiée aux gestes. Matériel et méthode: nous avons administré la batterie d'évaluation de la cognition et des praxies ainsi que deux tâches de mémoire de travail à 23 patients ayant été diagnostiqués avec la variante frontale de la démence fronto-temporale. Résultats: Nous trouvons une corrélation significative entre la performance au test des praxies et les mesures de mémoire de travail. Conclusions: Ces résultent confirment l'importance de la mémoire de travail dans la production des praxies and indiquent la possibilité d'une mémoire de travail spécifique aux gestes.

Mots-clefs: Apraxia; variante frontale de la démence frontotemporale; mémoire de travail; mémoire visuelle; mémoire gestuelle.

\section{Resumo}

\begin{abstract}
A literatura vem apontando que um déficit de memória de trabalho pode prejudicar a performance práxica. Este estudo visou a avaliar a presença de aparaxia em pacientes com a variante frontal da demência frontotemporal, examinar a ocorrência de correlações entre apraxia e memória de trabalho, e procurar evidências de uma memória de trabalho específica para gestos. Materiais e métodos: Vinte e três pacientes diagnosticados como tendo a variante frontal da demência frontotemporal foram examinados com uma bateria de avaliação cognitiva de praxias e com dois testes de memória de trabalho. Resultados: Correlações significativas entre a performance na bateria de praxia e nos testes de memória de trabalho. A performance na memória de trabalho gestual mostrou-se dissociada daquela visual e verbal. Conclusões: Tais resultados corroboram a importância da memória de trabalho na produção práxica e salientam a possibilidade de uma memória de trabalho específica para gestos.

Palavras-chave: Apraxia; demência frontotemporal; memória de trabalho; memória visual; memória gestual.
\end{abstract}

Artículo recibido: 11/15/2011; Artículo revisado: 24/08/2011; Artículo aceptado: 30/08/2011.

Pablo G. Gómez, Hospital Interzonal General de Agudos Eva Perón, San Martín, Provincia de Buenos Aires, CONICET. Daniel G. Politis, Hospital Interzonal General de Agudos Eva Perón, San Martín, Provincia de Buenos Aires, CONICET.

Correspondencia relacionada con este artículo debe ser enviada a: Pablo Gómez, Rosario77, C1424CCA, Ciudad Autónoma de Buenos Aires,

Argentina.

E-mail: pabloguillermogomez@yahoo.com.ar

DOI: $10.5579 / \mathrm{rnl} .2011 .0066$

Revista Neuropsicologia Latinoamericana 
It has been reported in the literature that a deficit in working memory may affect praxis performance. The aims of this study are to evaluate the presence of apraxia in patients with the frontal variant of frontotemporal dementia, to assess the occurrence of correlations between apraxia and working memory, and to look for evidence of a working memory specific for gestures. Material and methods: We administered to 23 patients diagnosed as having the frontal variant of frontotemporal dementia the praxis cognitive assessment battery and two tests of working memory. Results: We found significant correlations between the performance on praxis tests and the measures of working memory. The performance on gestural working memory was dissociated from that in visual and verbal working memory. Conclusions: These results reaffirm the importance of working memory in the production of praxis and point to the possibility of a working memory specific for gestures.

Keywords: Apraxia; frontal variant of frontotemporal dementia; working memory; visual memory; gestural memory.

La demencia frontotemporal (DFT) es el segundo tipo de demencia en frecuencia dentro de las demencia preseniles (Sjögren \& Andersen, 2005). Su característica más saliente es una profunda alteración del carácter y la conducta social, en el contexto de una conservación relativa de la percepción, las habilidades espaciales y la memoria. Se lo considera un síndrome de amplitud fenomenológica, en el que se distinguen tres grandes grupos clínicos: demencia frontotemporal variante frontal (DFTvf), demencia semántica y afasia progresiva no fluente (Neary, Snowden, Northen \& Goulding, 1988; Snowden, Neary \& Mann, 2002). En general, se acepta que uno de los elementos clínicos claves de la DFTvf es la relativa preservación de la memoria verbal y visual (Grossberg \& Desai, 2006; Neary et al., 1988; Snowden et al. 2002). Los errores de memoria son atribuidos a alteraciones ejecutivas antes que a una amnesia primaria (Neary, Snowden \& Mann, 2000).

La apraxia ha sido definida como un amplio espectro de alteraciones motoras de alto nivel que afecta el rendimiento de movimientos hábiles y/o aprendidos de los miembros superiores, como consecuencia de una lesión cerebral adquirida (Leiguarda \& Marsden, 2000). Ha sido mencionada en descripciones clínicas tempranas de la DFT (Pick, 1906). Asimismo, se ha adjudicado a los lóbulos frontales un rol de importancia en el control del sistema de praxias (Haaland, Harrington \& Knight, 2000; Heilman, González Rothi \& Valenstein, 1982). Pese a ello, aún no se han estudiado en forma sistemática las alteraciones práxicas en una población de paciente con DFTvf.

Baddeley \& Hitch (1974) propusieron la memoria de trabajo (MT) como un sistema de tres componentes: un ejecutivo central con una capacidad limitada de control atencional $\sin$ capacidad de almacenamiento propia, que controla dos subsistemas: un bucle articulatorio que opera con información acústico-verbal, y una agenda visuoespacial encargada del procesamiento de información visual y espacial. Más recientemente, Baddeley (2000), propuso como un cuarto componente al reten episódico, con la capacidad de mantener, manipular e integrar información de los demás sistemas subsidiarios y la memoria a largo plazo, llevando adelante algunas de las funciones atribuidas originalmente de manera implícita al ejecutivo central (Baddeley, 2002). Existe abundante evidencia de que la alteración del control ejecutivo está asociada al daño en los lóbulos frontales (Shallice, 1982, 1988). Estudios clínicos y de neuroimagen sugieren que el bucle articulatorio está asociado a regiones parietales y frontales (áreas de Brodmann 40 y 44) (Baddeley, 2000). La evidencia proveniente de la neuropsicología y las imágenes funcionales señalan a la agenda visuoespacial principalmente representada en el hemisferio derecho, como un sistema dependiente de la activación de los lóbulos occipitales (área 19) reflejando el componente visual, participación de áreas parietales (área 40) representando aspectos espaciales, y activación frontal (área 6 y 47) como responsable de la coordinación y el control (Smith \& Jonides, 1996). Asimismo, se ha señalado que el retén episódico no reflejaría el funcionamiento de una única región anatómica, pero parecería que las áreas frontales estarían profundamente involucradas en su funcionamiento (Baddeley, 2000).

En contraste con el bucle articulatorio, el dominio visuoespacial de la memoria de trabajo no tiene una estructura clara. La relación entre la memoria de trabajo espacial y visual, y las relaciones entre el almacenamiento sensorial y los procesos motores, aún no están dilucidadas (Wilson \& Emmorey, 1997)

En cuanto a la relación entre apraxia y MT, se sostiene que al incrementar los requerimientos de MT se dificulta la ejecución de gestos en pacientes con lesiones frontales. Se ha postulado que ejecutar una acción a la orden verbal implica necesariamente la planificación y la ejecución de esa acción, involucrando a la MT (Logie, Engelkamp, Dehn \& Rudkin, 2001).

Varios estudios han reportado interferencias en la MT visual, cuando en la fase de retención se ejecutan tareas motoras concurrentes no relacionadas (por ejemplo, Baddeley, Thomson \& Buchanan, 1975; Quinn \& Ralston, 1986; Smyth, Pearson \& Pendleton, 1988), ya sean estas movimientos oculares (Hale, Myerson, Rhee, Weiss \& Abrams, 1996) o de los miembros superiores (Lawrence, Myerson, Oonk \& Abrams, 2001). Por ejemplo, Lawrence et al. (2001) hallaron que la instrucción de presionar una botonera durante una tarea de memoria visual similar a la tareas de span de memoria visual de Wechsler (Wechsler, 1997) generaba interferencia sobre esta segunda tarea. A su vez, hay también evidencia de una facilitación en el recuerdo en tareas de MT espacial, evaluada por medio de tareas de tapping -como los cubos de Corsi (Milner, 1986), la tarea de span visual de Wechsler (Wechsler, 1997) o la prueba de atención visual de la batería de Artiola (Artiola, Hermosillo, Heaton y Pardee, 1999)cuando se ejecuta una tarea motora relacionada (Chum, Bekkering, Dodd \& Pratt, 2007), pero solo cuando la carga no excedía la capacidad máxima de la memoria de trabajo espacial (Luck \& Vogel, 1997). Por ejemplo, los sujetos instruidos a recordar la localización espacial de ítems presentados en la pantalla, mejoraban su rendimiento si al mismo tiempo se les permitía señalar sobre la pantalla cuando estos ítems se presentaban (Chum et al., 2007). Esta ventaja es atribuida a una mejora de las representaciones espaciales por el acto de señalar, a la formación de una representación espacial egocéntrica -entendida como una forma de codificar 
información espacial centrándose en los propios movimientos del cuerpo-, o a una combinación de ambas estrategias, sugiriendo la incorporación de conceptos de acción en el campo de la MT (Chum et al., 2007).

Halsband et al. (2001) hallaron que en las pruebas que exigían el uso de una sola mano, los pacientes con daño cerebral en la corteza premotora no diferían en comprensión y la imitación de gestos de los sujetos del grupo control, mientras que en las pruebas bimanuales los pacientes con lesiones frontales mostraban un severo déficit.

Asimismo, el deterioro específico de la MT en un paciente con lesiones isquémicas en el hemisferio izquierdo, demostrado por alteración en tareas duales, fue asociado con un déficit en la producción de pantomimas a partir de estimules verbales, visuales, táctiles y por imitación (Bartolo, Cubelli, Della Sala \& Dreib, 2003). Las tareas duales se emplean tradicionalmente para valorar la función de coordinación de la MT (Baddeley, 1996).

Cubelli, Marchetti, Boscolo \& Della Sala (2000) añaden un retén gestual al modelo de praxias de González Rothi, Ochipa \& Heilman (1991, 1997), siendo éste un componente de memoria a corto plazo, que sostiene los programas motores a ser ejecutados. Cubelli et al. (2000) sostienen que un déficit en el retén gestual daría lugar a una alteración en la producción de gestos tanto a la orden como a la imitación, afectando mayormente a las secuencias complejas por sobre los gestos simples, y con relativa conservación de la habilidad para distinguir o clasificar gestos.

Toraldo, Reverberi \& Rumiati (2001) postulan que, de estar alterada la MT en pacientes apráxicos con lesiones vasculares en el hemisferio izquierdo, debían afectarse más las secuencias de gestos por sobre las posiciones. Puesto que una alteración de la MT disminuiría la capacidad de almacenamiento, los gestos que impliquen una mayor cantidad de pasos van a ser los más afectados. En este sentido, un paciente con alteraciones en la MT debería rendir peor cuando los gestos a ejecutar impliquen varios pasos con un orden preciso (por ejemplo, el gesto de utilizar un sacacorchos), mientras que el desempeño en la producción de gestos estáticos (por ejemplo, pedir silencio) se mantendría relativamente conservado. Suponiendo constantes en cada paciente los recursos del praxicón de salida y la capacidad de programación motora, la razón entre los aciertos en la ejecución de secuencias por sobre los aciertos en la ejecución de posiciones (razón s/p) arrojaría un índice de los recursos de MT disponibles. Toraldo, Reverberi \& Rumiati (2001) concluyen que el grupo de pacientes vasculares tiene alteraciones en una MT gestual en relación con el grupo control.

En este mismo sentido, Bartolo et al. (2003) postulan la intervención en el procesamiento de praxias de la MT, entendida como un espacio de trabajo que integra y sintetiza las aferencias perceptuales con la información disponible del sistema de semántico de acción y los programas procedurales del praxicón de salida. Recordemos que así como los lexicones del modelo de lenguaje guardan la forma de las palabras conocidas, el praxicón de salida es el módulo del sistema de praxias que contiene los patrones de ejecución aprendidos (González Rothi, Ochipa \& Heilman, 1991, 1997). Rumiati, Zanini, Vorano y Shallice (2001) evaluaron pacientes apráxicos con lesiones en el hemisferio izquierdo y propusieron una forma de apraxia ideatoria, basada en el modelo de Norman y Shallice (1980/1986). Este modelo

Revista Neuropsicologia Latinoamericana (2011), 3(2), 23-30 teórico de atención postula que la selección de operaciones de rutina es descentralizada, a cargo del planificador de disputas, mientras que la selección de respuestas no rutinarias es cualitativamente diferente e involucra un sistema supervisor que modula el funcionamiento del sistema. A partir de este modelo, se interpreta que un déficit selectivo en el planificador de disputas provocaría una dificultad para acceder al repertorio de las características funcionales de los objetos, con conservación de la capacidad de secuenciar acciones.

El objetivo de este estudio es evaluar la presencia de apraxia en pacientes con DFTvf, así como la presencia de correlaciones entre apraxia y MT en este mismo grupo de pacientes. Adicionalmente, este trabajo busca encontrar evidencia de la existencia de una memoria de trabajo específica para gestos (Toraldo et al. 2001), a partir de disociaciones entre medidas de memoria de trabajo verbal, visual y gestual.

\section{Metodo}

\section{Participantes}

Fueron examinados 23 pacientes del Servicio de Neurología del Hospital Interzonal General de Agudos Eva Perón con diagnóstico de DFTvf de acuerdo a los criterios de Lund y Manchester (1994) y Neary et al. (1998). Los pacientes presentaron pérdida del tacto social, alteraciones en tareas de cognición social, rigidez, perseveraciones, anosognosia, fallas en tareas típicamente frontales como el test de ordenamiento de cartas de Wisconsin (Heaton, Chelune, Talley, Kay \& Curtis, 1993) o el Trail Making serie B (Reitan \& Wolfson, 1985). La instalación del cuadro fue insidiosa y progresiva, sin vinculación a lesiones traumáticas o enfermedad vascular. La totalidad de los pacientes presentó SPECT patológico, con predominio frontotemporal. Todos los pacientes presentaron alteraciones en al menos una prueba de cognición social.

La edad promedio fue de 67,7 años (DE 8,85) y la escolaridad de 7,8 años (DE 3,30). El 39,1\% fueron hombres y el $60,9 \%$ mujeres. Todos los pacientes fueron evaluados con un examen neurológico, estudios de neuroimagen y una extensa batería neuropsicológica que incluye la evaluación de la memoria verbal y visual, el lenguaje, la visuoconstrucción, la atención y las funciones ejecutivas. El examen neurológico no reflejó la presencia de signos extrapiramidales, síndrome extrapiramidal, síndrome piramidal, síndrome pseudobulbar, síndrome cerebeloso, apraxia de la marcha, sensibilidad o debilidad. El SPECT mostró alteraciones a nivel frontal en el $75 \%$ de los pacientes, alteraciones temporales en el $79 \%$ de los pacientes y alteraciones parietales en el 33\% de los pacientes. La evaluación incluyó las siguientes pruebas: California Verbal Learning Test, Memoria Lógica, Dígitos Directos e Inversos y Fluencia Verbal Fonológica de la Batería Neuropsicológica Española (Artiola et al., 1999); Figura Compleja de Rey (Meyers \& Meyers, 1995); Test de denominación por confrontación visual de Boston (Goodglass \& Kaplan, 1996); Fluencia verbal semántica (Butman, Allegri, Harris \& Drake, 2000); Trail Making Test A y B (Reitan \& Wolfson, 1985); subescalas de Analogía, Matrices, Vocabulario y Cubos de la Escala de Inteligencia para Adultos, WAIS III (Wechsler, 2002), Test de ordenamiento de cartas de Wisconsin (Heaton et al., 1993). Se evaluó cognición social mediante la prueba de Lectura de la Mente 
en los Ojos (Baron-Cohen, Wheelwright, Hill, Raste \& Plumb, 2001), Faux Pas (Stone, Baron-Cohen \& Knight, 1998) y Falsa Creencia de Primer Orden (Wimmer \& Perner, 1983).

Se utilizaron como grupo de comparación 14 sujetos normales para establecer el puntaje de corte de la razón $\mathrm{s} / \mathrm{p}$, estableciéndose el mismo a una desviación estándar y media por debajo de la media. En el grupo de comparación la edad promedio fue de 57,7 años (DE 13,86) y la escolaridad de 10,2 años (DE 4,08). No presentan diferencias respecto de la edad (U de Mann-Whitney 92,500, Z -2,150; p 0,032) ni la escolaridad (U de Mann-Whitney 109,500, Z -1,637; p 0,102) con el grupo de pacientes DFTvf.

Material empleado

Se administró la batería cognitiva de evaluación de praxias (Politis, 2003), la prueba de atención visual inversa de la batería neuropsicológica (Artiola et al., 1999) y la prueba de dígitos inversos del WAIS III (Wechsler, 1997).

La batería de evaluación de praxias comprende las siguientes pruebas: 1) Ejecución de gestos a la orden verbal; 2) Ejecución de gestos a la vista de objetos; 3) Uso de herramientas; 4) Discriminación gestual; 5) Decisión gestual; 6) Imitación de gestos familiares; 7) Apareamiento objetoherramienta; 8) Denominación por función; 9) Evaluación del conocimiento de una función por identificación; 10) Imitación de gestos no familiares. En cada prueba, el desempeño del paciente fue valorado como alterado (1) o conservado (0) de acuerdo al puntaje de corte de cada subescala de la batería.

Para la construcción de la razón s/p se seleccionaron los 7 gestos de la batería cognitiva de praxias que implican posiciones fijas o posturas (de aquí en adelante llamados posiciones) y los 10 gestos que involucran secuencias complejas que requieren dos o más movimientos distintos para su ejecución (de aquí en adelante, secuencias). Los gestos fueron extraídos de las siguientes pruebas: ejecución de gestos a la orden verbal, imitación de gestos familiares, e imitación de gestos no familiares. Cada uno de los gestos fue considerado como correcto o incorrecto, de acuerdo a los mismos criterios de la batería. La razón $\mathrm{s} / \mathrm{p}$ se calculó finalmente dividiendo el porcentaje de aciertos en la ejecución de gestos que implican secuencias (pe. llamar por teléfono) por el porcentaje de aciertos en la producción de gestos que implican posiciones (pe. gesto de silencio). Por ejemplo, si un paciente efectuaba correctamente la totalidad de los gestos estáticos (100\%), pero fallaba en la mitad de los gestos que implicaba una secuencia (50\%), el coeficiente s/p será de 0,5. Se estimará significativamente alterada en un paciente la razón $\mathrm{s} / \mathrm{p}$ al encontrarse un desvío y medio por debajo de la media del grupo de comparación.

La prueba de atención visual de la batería neuropsicológica (Artiola et al., 1999) es una prueba de atención visual y espacial (Lezak, 1995). Se le presenta al examinado un tablero con una serie de cubos, dispuestos de forma irregular. El examinado debe repetir manualmente secuencias de toques sobre los cubos, previamente realizadas por el examinador. La longitud de las secuencias crece progresivamente conforme el examinado reproduce correctamente las posiciones espaciales del examinado. En la sección denominada inversa, el examinado debe imitar en orden inverso, empezando por el último cubo de la secuencia, las posiciones demostradas por el examinador. Las tareas de tapping son tradicionalmente consideradas como medidas de memoria a corto plazo visoespacial (Smyth \& Scholey, 1992;
Baddeley, 2002), siendo necesaria la memoria de trabajo cuando la secuencia de posiciones debe ser reproducida en forma inversa (Vandierendonck, Kemps, Fastame \& Szmalec, 2004). Aunque es difícil encontrar tareas que se correspondan en forma pura con uno de los componentes de memoria de trabajo, se sugiere una asociación entre memoria de trabajo visuoespacial y tareas de tapping (Baddeley, 2002).

En la prueba de dígitos inversos del WAIS III (Wechsler, 1997) el examinado tiene que repetir secuencias de dígitos presentadas oralmente por el examinador. Las secuencias aumentan progresivamente su longitud. En la versión de dígitos denominada inversa, el examinado tiene que repetir las secuencias de dígitos en orden inverso al originalmente presentado.

El desempeño en las pruebas de atención visual inversa y dígitos inversos se considerará como medida de MT visual y MT verbal, respectivamente. Siguiendo a Toraldo et al. (2001) se examinará a la razón s/p como medida tentativa de MT gestual.

Procedimiento

Se utilizó el coeficiente de correlación de Pearson para valorar el nivel de correlación entre las diferentes pruebas y una $U$ de Mann-Whitney para verificar la presencia de diferencias significativas entre los pacientes con DFTvf y el grupo de comparación. Se utilizó un nivel alfa de 0,01 para todas las pruebas estadísticas.

\section{Resultados}

En un análisis a nivel de grupo, se halló que la totalidad de los pacientes evaluados mostraron alteraciones en al menos una de las pruebas de la batería de praxias. La Tabla I muestra los resultados de cada paciente en cada una de las pruebas de la batería. El 26,1 \% de los pacientes presentó alteraciones en la prueba de atención visual inversa, el 30,4\% presentó alteraciones en la prueba de dígitos inversos y el $34,8 \%$ mostró alteraciones en la razón s/p. Consideradas en conjunto, el $52,2 \%$ de los pacientes mostró alteraciones en al menos una medida de MT.

Se hallaron correlaciones significativas a nivel de grupo entre las pruebas de la batería de praxias, el puntaje total de la batería y las medidas de MT visual y gestual. La razón s/p correlaciona con el desempeño en 8 pruebas de la batería de praxias y con el puntaje total de la batería, pero sin embargo no correlaciona con las pruebas de atención visual inversa y dígitos inversos. La tabla II resume los resultados obtenidos.

La prueba $U$ de Mann-Whitney señaló diferencias significativas entre el grupo DFTfv y el grupo de comparación en el porcentaje de aciertos en secuencias y la razón s/p. El grupo DFTvf presentó un peor rendimiento en la producción de secuencias y un menor cociente en la razón s/p. No se encontraron diferencias entre ambos grupos en el porcentaje de aciertos en la producción de posiciones (Tabla III).

Con el objetivo de aportar evidencia a favor de la existencia de una MT específica para gestos, se buscaron disociaciones en el rendimiento individual de los pacientes del grupo DFTvf. Dos pacientes (casos 4 y 17) mostraron alteraciones selectivas en la razón $\mathrm{s} / \mathrm{p}$, con un rendimiento conservado en atención visual inversa y dígitos inversos. Un paciente (caso 11) mostró una alteración exclusiva en la prueba de atención visual inversa, con resultados normales en las pruebas de dígitos inversos y la razón s/p. Dos pacientes 
Tabla 1

Resumen del rendimiento del grupo de demencia frontotemporal variante frontal en las pruebas de la batería de praxias y los índices de memoria de trabajo

\begin{tabular}{|c|c|c|c|c|c|c|c|c|c|c|c|c|c|c|}
\hline & IAV & IVO & $\begin{array}{l}\text { Uso } \\
\text { Her } \\
\end{array}$ & Discr & Decis & $\begin{array}{c}\text { Imi } \\
\text { Fam } \\
\end{array}$ & $\mathrm{AOH}$ & $\begin{array}{l}\text { Den } \\
\text { Fun } \\
\end{array}$ & $\begin{array}{l}\text { Con } \\
\text { Fun } \\
\end{array}$ & $\begin{array}{c}\text { ImiNo } \\
\text { Fam }\end{array}$ & $\begin{array}{c}\text { Total } \\
\text { PX }\end{array}$ & AVI & DI & $\begin{array}{c}\text { Razon } \\
\mathrm{s} / \mathrm{p}\end{array}$ \\
\hline 1 & $* 10$ & $* 9$ & 2 & $* 5$ & $* 7$ & $* 4$ & $* 11$ & 0 & 0 & $* 8$ & 56 & $-0,923$ & $*_{-}-2,600$ & $*_{-1,770}$ \\
\hline 2 & $* 7$ & $* 5$ & 2 & 0 & 1 & $* 1$ & 2 & $* 2$ & 0 & 2 & 22 & $-0,538$ & 0,300 & $-0,669$ \\
\hline 3 & $* 9$ & $* 5$ & $* 3$ & $* 2$ & 2 & $* 2$ & 2 & $* 4$ & 0 & 3 & 32 & $-0,538$ & 0,300 & $-1,330$ \\
\hline 4 & $* 7$ & $* 7$ & 0 & $* 1$ & 0 & $* 2$ & 2 & 0 & 0 & 2 & 21 & $-0,538$ & 0,300 & $*-1,550$ \\
\hline 5 & 6 & $* 3$ & 1 & 0 & 3 & $* 2$ & 0 & 0 & 0 & 0 & 15 & $-0,154$ & 0,200 & $-0,890$ \\
\hline 6 & $* 11$ & $* 7$ & 1 & $* 3$ & 0 & $* 7$ & $* 6$ & $* 1$ & 0 & $* 7$ & 43 & $*-1,692$ & $*-2,200$ & $-0,596$ \\
\hline 7 & $* 8$ & $* 6$ & 1 & 0 & 0 & 0 & 0 & 0 & 0 & 1 & 16 & $-0,154$ & $*_{-} 2,000$ & $-0,596$ \\
\hline 8 & 3 & $* 3$ & 1 & 0 & 1 & $* 2$ & 3 & $* 2$ & 0 & 3 & 18 & $-1,308$ & 0,700 & $-0,449$ \\
\hline 9 & 5 & $* 3$ & 0 & 0 & 3 & $* 1$ & 1 & 0 & 0 & 4 & 17 & $-0,538$ & $-0,300$ & $-0,890$ \\
\hline 10 & 4 & $* 4$ & 0 & 0 & 1 & 0 & 0 & 0 & 0 & 1 & 6 & $-0,538$ & $-0,900$ & $-0,669$ \\
\hline 11 & $* 14$ & $* 16$ & $* 3$ & $* 5$ & $* 7$ & $* 5$ & 1 & $* 1$ & 0 & $* 8$ & 60 & *-1,692 & $-0,900$ & $-1,110$ \\
\hline 12 & $* 11$ & $* 9$ & 0 & $* 1$ & 3 & $* 3$ & 3 & $* 1$ & 0 & $* 7$ & 38 & $-0,923$ & $*_{-1,900}$ & $*_{-1,623}$ \\
\hline 13 & 6 & $* 7$ & $* 3$ & $* 1$ & $* 7$ & *6 & 2 & $* 1$ & 0 & $* 7$ & 8 & 0,231 & 0,000 & $-1,110$ \\
\hline 14 & 3 & $* 3$ & 0 & 0 & 2 & 0 & 1 & 0 & 0 & 3 & 12 & $-0,923$ & $*_{-} 1,900$ & $-0,890$ \\
\hline 15 & 6 & $* 4$ & 1 & 0 & 1 & 0 & 0 & $* 1$ & 0 & 1 & 14 & $-0,538$ & $-0,400$ & 0,123 \\
\hline 16 & $* 13$ & $* 10$ & $* 4$ & $* 3$ & $* 6$ & *6 & $* 12$ & $* 2$ & 0 & $* 9$ & 65 & $*-1,692$ & $*-1,700$ & *-1,880 \\
\hline 17 & $* 12$ & $* 11$ & $* 5$ & $* 2$ & $* 9$ & $* 6$ & $* 5$ & $* 1$ & 0 & $* 7$ & 58 & $-0,538$ & $-1,000$ & *-1,623 \\
\hline 18 & $* 16$ & $* 25$ & $* 10$ & $* 22$ & $* 20$ & $* 15$ & $* 18$ & $* 28$ & $* 20$ & *22 & 196 & $*-2,462$ & $*-2,100$ & $*_{-} 2,211$ \\
\hline 19 & $* 8$ & $* 7$ & 0 & $* 3$ & $* 8$ & $* 2$ & 2 & $* 1$ & 0 & 3 & 34 & $-0,154$ & 0,500 & $-1,330$ \\
\hline 20 & $* 23$ & $* 22$ & $* 5$ & $* 6$ & $* 14$ & $* 13$ & $* 7$ & $* 5$ & 0 & $* 20$ & 115 & $*-2,077$ & 0,400 & $*_{-1,880}$ \\
\hline 21 & $* 7$ & $* 10$ & 0 & 0 & 1 & $* 3$ & 0 & $* 1$ & 0 & $* 6$ & 28 & $*-1,692$ & $-1,200$ & $*_{-} 1,550$ \\
\hline 22 & 5 & 2 & 2 & 0 & 1 & 0 & 0 & $* 1$ & 0 & 1 & 12 & 0,615 & 0,600 & $-0,890$ \\
\hline 23 & $* 9$ & $* 3$ & 2 & $* 1$ & $* 7$ & $* 3$ & 4 & $* 2$ & 0 & $* 6$ & 37 & $-0,538$ & 0,600 & $-1,110$ \\
\hline
\end{tabular}

Nota. Los valores de las pruebas de la batería de praxias de presentan en puntaje bruto. Los índices de memoria de trabajo se presentan en puntaje $Z$.

IAV: Ingreso auditivo verbal; IVO: Ingreso visual de objetos; Uso Her: Uso de herramientas; Discr: Discriminación gestual; Decis: Decisión gestual; Imi Fam: Imitación de gestos familiares; AOH: Asociación objeto-herramienta; Den Fun: Denominación por función; Con Fun: Conocimiento de una función; Imi No Fam: Imitación de gestos no familiares; Total PX: Puntaje total de la batería de praxias; AVI: Atención visual inversa; DI: Dígitos inversos; Razón s/p: razón secuencias sobre posiciones. *Prueba significativamente alterada. 
Tabla 2

Correlaciones en demencia frontotemporal variante frontal entre los índices de memoria de trabajo y las pruebas de praxias

\begin{tabular}{ccccc}
\hline & & $\begin{array}{c}\text { Razón secuencias } \\
\text { sobre posiciones }\end{array}$ & $\begin{array}{c}\text { Atención visual } \\
\text { inversa }\end{array}$ & $\begin{array}{c}\text { Dígitos } \\
\text { inversos }\end{array}$ \\
\hline Razón secuencias sobre posiciones & $\mathrm{r}$ & & 0,441 & 0,248 \\
Atención visual inversa & $\mathrm{r}$ & 0,441 & 0,431 & 0,431 \\
Dígitos inversos & $\mathrm{r}$ & 0,248 & \\
Ejecución de gestos a la orden & & & & \\
verbal & $\mathrm{r}$ & $-0,648^{* *}$ & $-0,640^{* *}$ & $-0,206$ \\
Ejecución de gestos a la vista de & & & & $-0,305$ \\
objetos & $\mathrm{r}$ & $-0,693^{* *}$ & $-0,736^{* *}$ & $-0,143$ \\
Uso de herramientas & $\mathrm{r}$ & $-0,556^{* *}$ & $-0,469$ & $-0,335$ \\
Discriminación gestual & $\mathrm{r}$ & $-0,588^{* *}$ & $-0,617^{* *}$ & $-0,111$ \\
Decisión gestual & $\mathrm{r}$ & $-0,682^{* *}$ & $-0,475$ & $-0,226$ \\
Imitación de gestos familiares & $\mathrm{r}$ & $-0,660^{* *}$ & $-0,701^{* *}$ & $-0,448$ \\
Asociación Objeto Herramienta & $\mathrm{r}$ & $-0,669^{* *}$ & $-0,630^{* *}$ & $-0,193$ \\
Denominación por función & $\mathrm{r}$ & $-0,459$ & $-0,529 * *$ & $-0,287$ \\
Conocimiento de una función & $\mathrm{r}$ & $-0,413$ & $-0,465$ & $-0,285$ \\
Imitación de gestos no familiares & $\mathrm{r}$ & $-0,702^{* *}$ & $-0,747^{* *}$ & $-0,291$ \\
\hline Puntaje total de la batería de praxias & $\mathrm{r}$ & $-0,688^{* *}$ & $-0,687^{* *}$ & \\
\hline
\end{tabular}

Nota. $* *$ La correlación es significativa al nivel 0,01

Tabla 3

Diferencias entre la población de pacientes con demencia frontotemporal variante frontal y el grupo de comparación en secuencias y posiciones

\begin{tabular}{|c|c|c|c|c|}
\hline & & $\begin{array}{c}\text { U de Mann- } \\
\text { Whitney }\end{array}$ & $\mathrm{Z}$ & $\mathrm{p}$ \\
\hline$\%$ de aciertos en secuencias gestuales & $\begin{array}{l}\text { NOR } 93,9 \% \\
\text { DFT } 61,30 \%\end{array}$ & 29,500 & $-4,1760$ & $000 * *$ \\
\hline$\%$ de aciertos en posiciones gestuales & $\begin{array}{l}\text { NOR } 85,7 \% \\
\text { DFT } 91,30 \%\end{array}$ & 153,000 & $-0,289$ & 0,817 \\
\hline $\begin{array}{l}\text { Razón entre los aciertos en la ejecución } \\
\text { de secuencias por sobre posiciones }\end{array}$ & $\begin{array}{ll}\text { NOR } & 1,204 \\
\text { DFT } & 0,681\end{array}$ & 33,000 & \multicolumn{2}{|c|}{$-4,0300,000 * *$} \\
\hline
\end{tabular}

Nota. $* *$ La diferencia es significativa al nivel 0,01

NOR: Controles normales.

DFT: Pacientes con demencia frontotemporal, variante frontal.

(caso 7 y 14) presentaron un déficit exclusivo en la prueba de dígitos inversos, sin alteraciones en las pruebas de atención visual inversa y razón s/p. En total, el índice de la razón s/p se mostró disociado del rendimiento en atención visual inversa en seis pacientes (casos 1, 4, 6, 11, 12 y 17) y del rendimiento en dígitos inversos en siete pacientes (casos 4, 6, 7, 14, 17, $20 \mathrm{y}$ 21). La tabla IV resume los resultados de los pacientes que mostraron alteraciones selectivas en estas pruebas.

\section{Discusión}

En primer lugar, este estudio aporta nueva evidencia de la presencia de alteraciones práxicas en DFTvf (Rubinstein y Politis, 2007) y replica el hallazgo de apraxia temprana en otros cuadros demenciales (Mozaz et al., 2006; TravniczekMarterer, Danielczyk, Simanyi \& Fischer, 1993; Willis,
Behrensb, Macka \& Chuia, 1998). Asimismo, también se halló que al incrementar la carga de memoria, los pacientes con lesiones frontales muestran mayores dificultades al momento de ejecutar gestos.

La observación de que solo la mitad de los pacientes evaluados presenten alteraciones en las pruebas de MT es consistente con el postulado que propone que en estadios iniciales, los pacientes con DFTvf manifiestan inicialmente alteraciones del comportamiento y la personalidad con relativa preservación de la cognición (Jenner, Reali, Puopolo \& Silveri, 2006; Snowden et al., 2002). En la evaluación neuropsicológica, éste déficit se expresaría principalmente en alteraciones de la cognición social (Cummings, 1993), especialmente en etapas iniciales del trastorno, cuando la MT 
Tabla 4

Resumen del rendimiento del grupo de demencia frontotemporal variante frontal en las pruebas de memoria de trabajo

\begin{tabular}{cccc}
\hline & AVI & DI & Razon s/p \\
\hline 4 & + & + & - \\
17 & + & + & - \\
11 & - & + & + \\
7 & + & - & + \\
14 & + & - & + \\
1 & + & - & - \\
6 & - & - & + \\
12 & + & - & - \\
20 & - & + & - \\
21 & - & + & - \\
\hline
\end{tabular}

Nota. AVI: Atención visual inversa; DI: Dígitos inversos; Razón s/p: razón secuencias sobre posiciones. + prueba conservada, - prueba alterada (1,5 DE por debajo de la media)

puede mostrarse normal (Rahman, Sahakian, Hodges, Rogers \& Robbins, 1999).

Los índices de MT visual y gestual no mostraron correlaciones significativas con la prueba que valora el uso de objetos, mientras que presentaron correlaciones elevadas con las pruebas ejecución de gestos a la orden verbal, ejecución de gestos a la vista de objetos, e imitación de gestos familiares y no familiares. Estos hallazgos son consistentes con el hecho de que la MT tendría una menor relevancia en el uso de objetos, mientras que sería clave para generar pantomimas (Bartolo et al., 2003).

El modelo original de memoria de trabajo de tres componentes planteado por Baddeley, compuesto por un ejecutivo central, un bucle articulatorio y una agenda visuoespacial, no permite explicar completamente las disociaciones encontradas. La doble disociación encontrada entre la alteración de la prueba de atención visual inversa (tomada como medida de MT visual) y la alteración de la razón s/p (postulada como medida de MT gestual) implicaría que ambos procesos son al menos parcialmente independientes. Asimismo, la doble disociación entre la alteración de la prueba de dígitos inversos (entendida como medida de MT verbal) y la alteración de la razón $\mathrm{s} / \mathrm{p}$, indicaría en el mismo sentido la relativa independencia de los procesos involucrados en ambas tareas. A nivel de grupo, encontramos que el desempeño en estas tres tareas diferentes no correlaciona en este grupo de pacientes. A su vez, a nivel de caso único, el rendimiento en estas tareas se encuentra doblemente disociado. Ambos resultados indicarían que la MT postulada para los modelos de praxias (Toraldo, Reverberi \& Rumiati, 2001; Bartolo et al., 2003) no podría interpretarse en términos de MT verbal o MT visuoespacial. Se ha postulado que la agenda visuoespacial puede trabajar a su vez con información motora o táctil, siendo un desafío para próximas investigaciones separar estos componentes de los dominios visuales y espaciales (Baddeley, 2002). La MT representada por el coeficiente $\mathrm{s} / \mathrm{p}$, que correlaciona estrechamente con las pruebas de praxias, podría tratarse de una forma de MT gestual o cinética.

\section{Conclusiones}

Estos datos apoyarían la existencia de una MT específica de gestos (Toraldo et al., 2001). El hallazgo de alteraciones significativas en la razón $\mathrm{s} / \mathrm{p}$ en pacientes apráxicos con DFTvf revelaría que la MT gestual se encuentra afectada en esta población.

\section{Referencias Bibliograficas}

Artiola, L., Hermosillo, D., Heaton, R. K. \& Pardee, R. E. (1999). Manual de normas y procedimientos para la Batería Neuropsicológica en Español. Tucson, Arizona: m Press.

Baddeley, A. D. (1996). Working memory. Quarterly Journal of Experimental Psychology, 49A, 5-28.

Baddeley, A. D. (2000). The episodic buffer: A new component of working memory? Trends in Cognitive Sciences, 4, 417423.

Baddeley, A. D. (2002). Is Working Memory Still Working? European Psychologist, 7, 85-97.

Baddeley, A. D. \& Hitch, G. J. (1974). Working memory. En G. A. Bower (Ed.), Recent advances in learning and motivation 8, (pp. 47-90). New York: Academic Press.

Baddeley, A. D., Thomson, N. \& Buchanan, M. (1975). Word length and the structure of short-term memory. Journal of Verbal Learning and Verbal Behavior, 14, 575-589.

Baron-Cohen, S., Wheelwright, S., Hill, J., Raste, Y. \& Plumb, I. (2001). The Reading the Mind in the Eyes ${ }^{\text {re }}$ Test Revised Version: A study with normal adults, and adults with Asperger Syndrome or High-Functioning Autism. The Journal of Child Psychology and Psychiatry, 42, 241-252.

Bartolo, A., Cubelli, R., Della Sala, S. \& Dreib, S. (2003). Pantomimes are special gestures which rely on working memory. Brain and Cognition, 53, 483-494.

Butman, J., Allegri, R. F., Harris, P. \& Drake, M. (2000). Fluencia verbal en español. Datos Normativos en Argentina. Medicina, 60, 561-564

Chum, M., Bekkering, H., Dodd, M. D. \& Pratt, J. (2007). Motor and visual codes interact to facilitate visuospatial memory performance. Psychonomic Bulletin \& Review, 14 (6), 1189-1193.

Cubelli, R., Marchetti, C., Boscolo, G. \& Della Sala, S. (2000). Cognition in action: Testing a model of limb apraxia. Brain and Cognition, 44, 144-165.

Cummings, J. L. (1993). Frontal-subcortical circuits and human behaviour. Archives of Neurology, 50, 873-80.

González Rothi, L. J., Ochipa, C., \& Heilman, K. M. (1991). A cognitive neuropsychological model of limb apraxia. Cognitive Neuropsychology, 8, 443-458.

González Rothi, L. J., Ochipa, C., \& Heilman, K. M. (1997). The neuropsychology of action. East Sussex: Psychology Press.

Goodglass, H. \& Kaplan, E. (1996). Evaluación de la afasia y de trastornos relacionados. 2nd ed. Madrid: Panamericana.

Grossberg, G. T. \& Desai, A. L. (2006). Cognition in Alzheimer's Disease and Related Disorders. En C. G. Kruse, H. Y. Meltzer, C. Sennef, S. V. Van De Witte. Thinking about Cognition: Concepts, Targets and Therapeutics (pp. 23). Michigan: IOS Press.

Haaland, K. Y., Harrington, D. L. \& Knight, R. T. (2000). Neural representations of skilled movement. Brain, 12, 2306-2313.

Hale, S., Myerson, J., Rhee, S. H., Weiss, C. S. \& Abrams, R. A. (1996). Selective interference with the maintenance of location information in working memory. Neuropsychology, 10, 228-240.

Halsband, U., Schmitt, J., Weyers, M., Binkofski, F., Grützner, G. \& Freund, H. J. (2001). Recognition and imitation of pantomimed motor acts after unilateral parietal and 
premotor lesions: A perspective on apraxia. Neuropsychologia, 39, 200-216.

Heaton, R. K., Chelune, G. J., Talley, J. L., Kay, G. G. \& Curtis, G. (1993). Wisconsin Card Sorting Test (WCST). Manual Revised and Expanded. Odessa, FL: Psychological Assessment Resources.

Heilman, K. M., Gonzalez Rothi, L. J. \& Valenstein, E. (1982). Two forms of ideomotor apraxia. American Academy of Neurology, 32, 342-346.

Jenner, C., Reali, G., Puopolo, M. \& Silveri, M. C. (2006). Can cognitive and behavioural disorders differentiate frontal variant-frontotemporal dementia from Alzheimer's disease at early stages?. Behavioural Neurology, 17, 89-95.

Lawrence, B. M., Myerson, J., Oonk, H. M. \& Abrams, R. A. (2001). The effects of eye and limb movements on working memory. Memory, 9, 433-444.

Leiguarda, R. C. \& Marsden, C. D. (2000). Limb apraxias: higherorder disorders of sensorimotor integration. Brain, 123, 860-879. Luck, S. J. \& Vogel, E. K. (1997). The capacity of visual working memory for features and conjunctions. Nature, 390, 279-281.

Lezak, M. D. (1995). Neuropsychological assessment ( $3^{\circ}$ ed.). New York: Oxford University Press.

Logie, R. H., Engelkamp, J., Dehn, D., \& Rudkin, S. (2001). Actions, mental actions, and working memory. En M. Denis, R. H. Logie, C. Cornoldi, M. DeVega, M. J. Engelkamp (Eds). Imagery, language and visuo-spatial thinking (pp. 161184). Hove: Psychology Press. Mega, M. S. \& Cummings, J. L. (1994). Frontal-subcortical circuits and neuropsychiatric disorders. The Journal of Neuropsychiatry and Clinical Neurosciences, 6, 358-370.

Luck, S. J. \& Vogel, E. K. (1997). The capacity of visual working memory for features and conjunctions. Nature, 390, 279281.

Meyers, J. E. \& Meyers, K. R. (1995). Rey Complex Figure Test and Recognition Trial. Professional Manual. Odessa, Florida: Psychological Assessment Resources.

Milner, B. (1986). Interhemispheric dipherences in the localization of psychological processes in man. British Medical Bulletin, 27, 272-277.

Mozaz, M., Garaigordobil, M., González Rothi, L. J., Anderson, J., Crucian, G. P. \& Heilman, K. M. (2006). Posture recognition in Alzheimer's disease. Brain and cognition, $62,241-245$

Neary, D., Snowden, J. S. \& Mann, D. M. A. (2000). Classification and description of frontotemporal dementias. Annals of the New York Academy of Sciences, 920, 46-51.

Neary, D., Snowden, J. S., Gustafson, L., Passant, U., Stuss, D., Black, S. et al. (1998). Frontotemporal lobar degeneration: A consensus on clinical diagnostic criteria. Neurology, 51, $1546-1554$

Neary, D., Snowden, J. S., Northen, B. \& Goulding, P. (1988). Dementia of frontal lobe type. Journal of Neurology, Neurosurgery, and Psychiatry, 51, 353-361.

Norman, D. A. \& Shallice, T. (1980). Attention to action: Willed and automatic control of behaviour. Center for Human Information Processing (Technical Report No. 99). Reprinted 1986 R. J. Davidson, G. E. Schwartz, D. Shapiro (Eds.) Consciousness and self regulation: Advances in Research and Theory, 4. New York: PlenumPress.

Pick A. (1906). Über einen weiteren Symptomenkomplex im Rahmen der Dementia senilis, bedingt durch umschriebene stärkere Hirnatrophie (gemischte Apraxie). [A través de otro grupo de síntomas en el contexto de la demencia senil, causada por atrofia cerebral circunscrita (Apraxia mixta)]. Monatsschrift fur Psychiatric and Neurologic, 19, 97-108.

Politis, D. (2003). Nuevas perspectivas en la evaluación de las apraxias. Tesis doctoral para la obtención del título de Doctor en Medicina, Facultad de Medicina, Universidad de Buenos Aires, Buenos Aires.
Quinn, J. G. \& Ralston, G. E. (1986). Movement and attention in visual working memory. Quarterly Journal of Experimental Psychology 38, 689-703.

Rahman, S., Sahakian, B. J., Hodges, J. R., Rogers, R. D. \& Robbins, T. W. (1999). Specific cognitive deficits in mild frontal variant frontotemporal dementia. Brain: a Journal of Neurology, 122, 1469-93.

Reitan, R. \& Wolfson, D. (1985). The Halstead-Reitan Neuropsychological Test Battery. Tucson, Arizona: Neuropsychology Press.

Rubinstein, W. Y. y Politis, G. D. (2007). Apraxia en diferentes tipos de demencia. $X$ Congreso Latinoamericano de Neuropsicología. Revista Argentina de Neuropsicología, 10, 22- 214.

Rumiati, R. I., Zanini, S., Vorano, L. \& Shallice, T. (2001). A form of ideational apraxia as a selective deficit of contention scheduling. Cognitive Neuropsychology, 18, 617-642.

Shallice, T. (1982) . Specific impairments of planning. Philosophical Transaction of the Royal Society London, B, 298, 199-209.

Shallice, T. (1988). From neuropsychology to mental structure. Cambridge: Cambridge University Press.

Sjögren, M. \& Andersen, C. (2006). Frontotemporal Dementia - A brief review. Mechanisms of ageing and development, 127, 180-187.

Smith, E. E. \& Jonides, J. (1996). Working memory in humans: Neuropsychological evidence. En M. Gazzaniga (Ed.), The cognitive neurosciences (pp. 1009-1020). Cambridge, MA: MIT Press.

Smyth, M. M., Pearson, N. A. \& Pendleton, L. R. (1988). Movement and working memory: patterns and positions in space. Quarterly Journal of Experimental Psychology, 41, 235250.

Smyth, M. M., \& Scholey, K. A. (1992). Determining spatial span: The role of movement time and articulation rate. Quarterly Journal of Experimental Psychology, 45A, 479-501.

Snowden, J. S., Neary, D. \& Mann, D. M. A. (2002). Frontotemporal dementia. The British journal of psychiatry: the journal of mental science, 180, 140-143.

Stone, V. E., Baron-Cohen, S. \& Knight, R. T. (1998). Frontal lobe contributions to theory of mind. Journal of Cognitive Neuroscience, 10 (5), 640-656.

The Lund and Manchester Groups. (1994). Clinical and neuropathological criteria for frontotemporal dementia. Journal of neurology, neurosurgery, and psychiatry, 57, 416-418.

Toraldo, A., Reverberi, C. \& Rumiati, R. I. (2001). Critical dimensions affecting imitation performance of patients with ideomotor apraxia. Cortex; a journal devoted to the study of the nervous system and behavior, 37, 737-740.

Travniczek-Marterer, A., Danielczyk, W., Simanyi, M. \& Fischer, P. (1998). Ideomotor apraxia in Alzheimer's disease. Acta Neurologica Scandinavica, 88, 1-4.

Vandierendonck, A., Kemps, E., Fastame, M. C. \& Szmalec, A. (2004). Working memory components of the Corsi blocks task. British Journal of Clinical Psychology, 95, 57-79.

Wechsler, D. (1997). Wechsler Adult Intelligences Scale-third edition. San Antonio, TX: The psychological corporation.

Wechsler, D. (2002). WAIS III: Test de inteligencia para adultos. Manual Técnico. Buenos Aires: Paidós.

Willis, L., Behrensb, M., Macka W. \& Chuia, H. (1998). Ideomotor Apraxia in Early Alzheimer's Disease: Time and Accuracy Measures. Brain and Cognition, 38, 220-233.

Wilson, M. \& Emmorey, K. (1997). A visuospatial "phonological loop" in working memory: Evidence from American Sign Language. Memory \& Cognition, 25, 313-320.

Wimmer, H. \& Perner, J. (1983). Beliefs about beliefs: Representation and constraining function of wrong beliefs in young children's understanding of deception. Cognition, 13 (1), 103-128. 